\title{
Liposarcoma masquerading as an inflammatory pseudotumor: a case report

\begin{abstract}
Background: Distinguishing an atypical lipomatous tumor/well-differentiated liposarcoma from a benign lipomatous tumor on morphology alone can be difficult and there is an established role for MDM2 fluorescent in situ hybridization studies in making this differential diagnosis. There is no literature on the role for MDM2 fluorescent in situ hybridization studies in distinguishing between a well-differentiated liposarcoma with extreme fibrosis and a fibrosing inflammatory pseudotumor.

Case presentation: We report the case of a 76-year-old Australian woman initially diagnosed by an excision biopsy with a retroperitoneal fibrosing inflammatory pseudotumor. She was then diagnosed 5 years later with a pleomorphic undifferentiated sarcoma. Upon review of the original resection specimen, we were able to show that the tumor demonstrated MDM2 amplification. MDM2 amplification was also present in some adjacent bland adipose tissue, and also in the tumor recurrence as a pleomorphic undifferentiated sarcoma.

Conclusion: Taken together, our findings provide strong evidence that the original tumor was a misdiagnosed welldifferentiated liposarcoma with extreme fibrosis, and the pleomorphic undifferentiated sarcoma represented a recurrence of the same tumor with dedifferentiation.
\end{abstract}

Keywords: Benign lipomatous tumor, Liposarcoma, MDM2

\section{Background}

The distinction between atypical lipomatous tumor/welldifferentiated liposarcoma (ALT/WDLPS) and benign lipomatous tumors can be difficult, and fluorescent in situ hybridization (FISH) studies for MDM2 amplification have an established role in the pathological differential diagnosis [1-8]. Distinguishing between WDLPS with extreme sclerosis and fibrosing inflammatory pseudotumor can be equally problematic, but FISH studies for MDM2 are infrequently used in this setting. We present a case of low grade liposarcoma initially misdiagnosed as a fibrosing retroperitoneal pseudotumor and discuss the utility of MDM2 FISH in establishing a definitive tissue diagnosis.

\footnotetext{
* Correspondence: affgill@med.usyd.edu.au

${ }^{1}$ Department of Anatomical Pathology, Royal North Shore Hospital, Pacific Highway, St Leonards, NSW 2065, Australia

${ }^{4}$ University of Sydney, Sydney, NSW 2006, Australia

Full list of author information is available at the end of the article
}

\section{Case presentation}

Our patient was a 76-year-old Caucasian Australian woman who originally presented over 5 years ago with urinary symptoms initially suggestive of recurrent urinary tract infection. At that time, she had an unremarkable past medical history of mild hypertension with no previous hospitalizations and did not smoke cigarettes. She underwent a work-up for the ongoing dysuria and urgency symptoms and imaging demonstrated a highdensity retroperitoneal tumor with some fat density around her right inguinal canal. This tumor was surgically resected. On gross examination, the retroperitoneal tumor was $10 \mathrm{~cm}$ in diameter with a lobulated contour and a pale whorled cut surface reminiscent of uterine leiomyoma. On histological examination, there was widespread, dense fibrosis, with a variable chronic inflammatory infiltrate rich in plasma cells that was most prominent at the periphery of the tumor (Fig. 1a). The tumor was hypocellular and densely fibrotic, composed predominantly of bland stromal cells with morphology suggestive of fibroblasts (Fig. 1b, c). Very occasional 


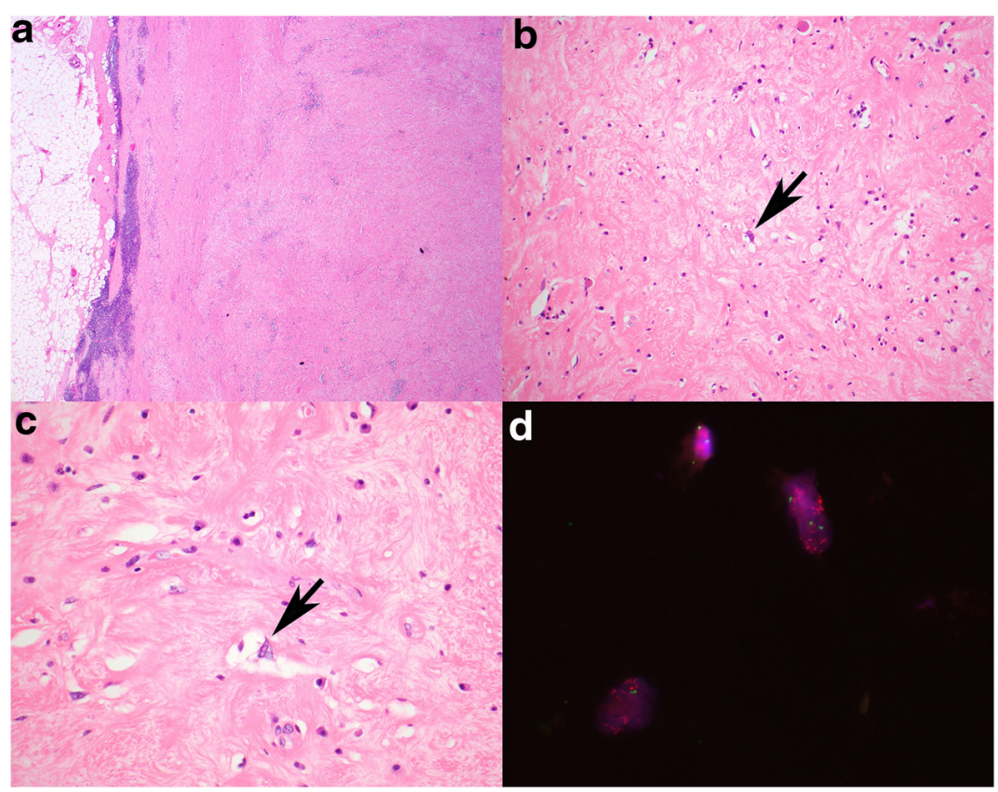

Fig. 1 Representative photomicrographs from the initial resection specimen. a The tumor was relatively well circumscribed, with scattered lymphoid aggregates noted at the edge. $\mathbf{b}$ There was extensive fibrosis with scattered inflammatory cells and occasional enlarged cells with vague vacuolation (arrow). c Upon review, some of these atypical cells (arrow) showed cytoplasmic vacuolation raising the possibility of a lipoblast. d Fluorescent in situ hybridization studies demonstrated amplification of MDM2 (red signal) compared to the chromosome 12 centromere probe (green signal). Original magnifications a 20x, b 100x, c 400x, d 1000x

mildly atypical larger cells were noted and some were vacuolated (Fig. 1b, c), but no diagnostic lipoblasts were identified. The fat from her right inguinal canal was composed of mature adipose tissue with extensive fat necrosis. Again, no lipoblasts were identified. Immunohistochemistry demonstrated that the tumor was negative for S100, CD34, cKit, desmin, and anaplastic lymphoma kinase (ALK), and there was no increase in immunoglobulin (Ig)G4-positive plasma cells. Although there was some uncertainty, the retroperitoneal tumor was diagnosed as an inflammatory pseudotumor in fibrosing stage (IgG4-negative variant). The area of fat necrosis in her right inguinal canal was considered benign.

Our patient re-presented 5 years later with similar urinary symptoms and imaging demonstrated a retroperitoneal tumor at the site of the previous excision. She underwent en block excision with a right hemicolectomy. The excised tumor was centered in the deep mesocolon. Again the tumor was lobulated and $10 \mathrm{~cm}$ in diameter with a somewhat pale fleshy cut surface. An incomplete rim of chronic inflammation was again noted (Fig. 2a) but the recurrent tumor was composed of poorly differentiated pleomorphic cells including bizarre forms (Fig. 2b, c). That is, the tumor demonstrated the morphology of a pleomorphic undifferentiated sarcoma. Despite the marked pleomorphism, mitoses were infrequent (less than 1 per 10 high-power fields). Again, occasional vacuolated cells were present (Fig. 2c) but classic lipoblasts were not identified.
Immunohistochemistry again demonstrated that the neoplastic cells were negative for S100, CD34, cKit, desmin, and ALK. Given the identical location and presence of a rim of inflammatory cells it was clear that the recurrence represented dedifferentiation of the primary

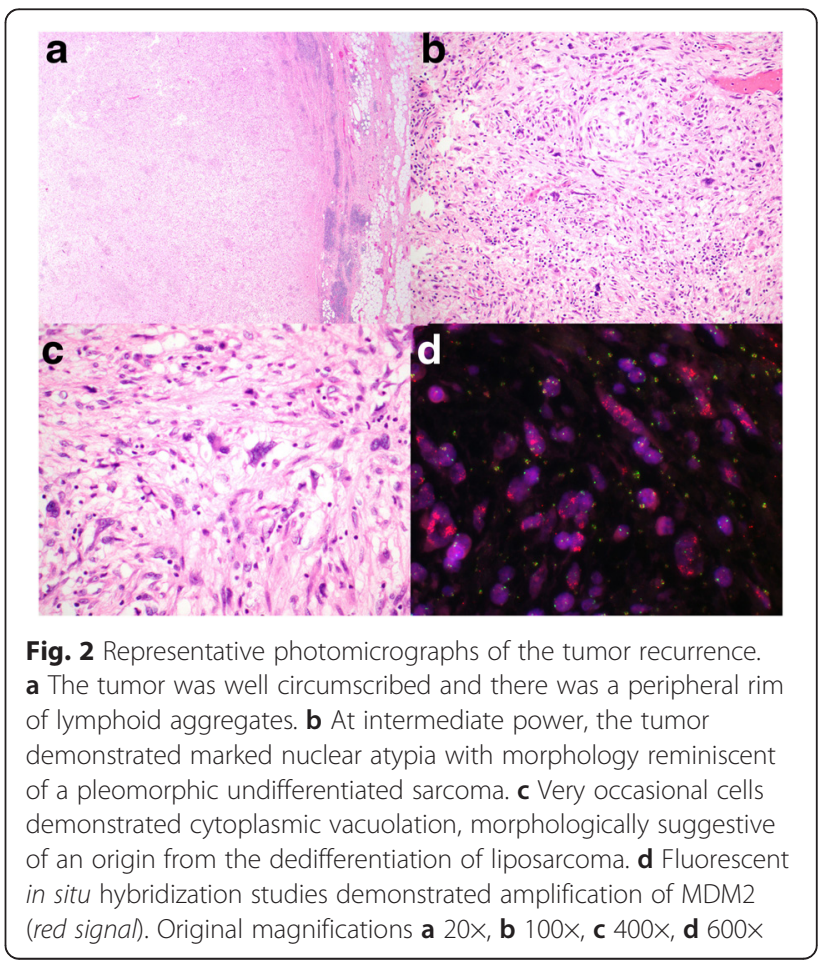


tumor. Upon review of both cases, the possibility of dedifferentiated liposarcoma was considered. The primary tumor, the bland adipose tissue resected from the inguinal canal at the time of surgery, and the recurrent tumor all underwent FISH testing, which demonstrated MDM2 amplification in all three specimens (Figs 1d and 2d). Therefore a final diagnosis of ALT/WDLPS with subsequent dedifferentiation and recurrence as a dedifferentiated liposarcoma was made.

\section{Discussion}

The demonstration of amplification of MDM2 with FISH has proven to be a robust and reliable method of differentiating ALT/WDLPS from benign lipomatous tumors [1-8]. For example, Kimura et al. found MDM2 amplification in $98 \%$ (48 of 49) of ALT/WDLPS but in no benign adipose tumors [1]. Similarly Weaver et al. demonstrated MDM2 amplification in $100 \%$ of ALT/ WDLPS (13 out of 13) and dedifferentiated liposarcomas (14 out of 14) [2]. Of note MDM2 amplification has been consistently demonstrated in areas of WDLPS with minimal cellular atypia, indicating that the presence of MDM2 amplification even in cytologically bland adipose tissue can be considered prima facie evidence of ALT/ WDLPS [2]. That is, if MDM2 FISH studies had been available and were performed prospectively in our case at first presentation in 2010, a diagnosis of ALT/WDLPS could have been justified despite the bland cytology.

There is also emerging evidence that the presence of MDM2 amplification in an otherwise undifferentiated sarcoma is strong evidence that the tumor has arisen from dedifferentiation of a liposarcoma. For example, in Le Guellec et al. [3] reported that tumors that would otherwise be classified as pleomorphic undifferentiated sarcoma, but that demonstrated MDM2 amplification, had similar clinical characteristics, morphology, genomic profile, and outcome to conventional dedifferentiated liposarcoma and were significantly less aggressive than pleomorphic undifferentiated sarcomas without MDM2 amplification. Our case, which provides clear evidence of a pleomorphic undifferentiated sarcoma arising from a lower grade tumor with MDM2 amplification, further supports this finding.

\section{Conclusion}

To exclude ALT/WDLPS, it has been recommended that MDM2 FISH studies be performed in any atypical adipose tumors, including any adipose tumor with equivocal cytological atypia or cytologically bland tumors with recurrence, large size, deep location, and/or retroperitoneal location [7]. This case demonstrates that there may also be a role for MDM2 studies to exclude a diagnosis of WDLPS with extreme sclerosis in patients apparently presenting with a retroperitoneal inflammatory pseudotumor.

\section{Consent}

Written informed consent was obtained from the patient for publication of this case report and accompanying images. A copy of the written consent is available for review by the Editor-in-Chief of this journal.

\section{Competing interests}

The authors declare that they have no competing interests.

\section{Authors' contributions}

CS and SO analyzed and interpreted the MDM2 FISH studies. JE interpreted our patient's clinical history. AG, RE, and JR performed the histological examination of the tumors and were major contributors in writing the manuscript. All authors read and approved the final manuscript.

\section{Author details}

${ }^{1}$ Department of Anatomical Pathology, Royal North Shore Hospital, Pacific Highway, St Leonards, NSW 2065, Australia. ${ }^{2}$ Tissue Pathology and Diagnostic Oncology, Royal Prince Alfred Hospital, Building 94 Missenden Road, Camperdown, NSW 2050, Australia. ${ }^{3}$ Department of Surgery, Royal North Shore Hospital, Pacific Highway, St Leonards, NSW 2065, Australia. ${ }^{4}$ University of Sydney, Sydney, NSW 2006, Australia.

Received: 22 July 2015 Accepted: 2 March 2016

Published online: 18 March 2016

\section{References}

1. Kimura H, Dobashi Y, Nojima T, et al. Utility of fluorescence in situ hybridization to detect MDM2 amplification in liposarcomas and their morphological mimics. Int J Clin Exp Pathol. 2013:6:1306-16.

2. Weaver J, Downs-Kelly E, Goldblum J, et al. Fluorescence in situ hybridization for MDM2 gene amplification as a diagnostic tool in lipomatous neoplasms. Mod Pathol. 2008;21:943-9.

3. Le Guellec S, Chibon F, Ouali M, et al. Are peripheral purely undifferentiated pleomorphic sarcomas with MDM2 amplification dedifferentiated liposarcoms? Am J Surg Pathol. 2014;38:293-304.

4. Sirvent N, Coindre J, Maire G, et al. Detection of MDM2-CDK4 amplification by fluorescence in situ hybridization in 200 paraffin-embedded tumour samples: utility in diagnosing adipocytic lesions and comparison with immunohistochemistry and real-time PCR. Am J Surg Pathol. 2007;31:1476-89.

5. Binh M, Sastre-Garau X, Guillou L, et al. MDM2 and CDK4 immunostainings are useful adjuncts in diagnosing well-differentiated and dedifferentiated liposarcoma subtypes: a comparative analysis of 559 soft tissue neoplasms with genetic data. Am J Surg Pathol. 2005;29:1340-7.

6. Marcarenco R, Erickson M, Wang X, et al. Retroperitoneal lipomatous tumours without cytologic atypia: are they lipomas? Am J Surg Pathol. 2009;33(10): 1470-6.

7. Zhang $\mathrm{H}$, Erickson-Johnson M, Wang W, et al. Molecular testing for lipomatous tumours: critical analysis and test recommendations based on the analysis of 405 extremity-based tumors. Am J Surg Pathol. 2010;34(9): 1304-11.

8. Weiss SW, Goldblum JR. Enzinger and Weiss's soft tissue tumours. 6th ed. St Louis: Mosby; 2014. 